\title{
Decisions about referrals for psychological therapies: a matched-patient qualitative study
}

\author{
Stavros Stavrou, John Cape and Chris Barker
}

\section{ABSTRACT}

Background

Psychological therapies are effective treatments for common mental health problems, but access is limited. GPs face difficult decisions as to whom to refer, but little is known about this decision-making process.

Aim

To explore GPs' accounts of decisions to refer, or not refer, patients for psychological therapy.

Design of study

A qualitative study, using a matched-patient procedure. Setting

General practices in two inner London boroughs.

Method

In semi-structured interviews, GPs were asked to compare and contrast five matched-patient pairs, consisting of patients who had been referred for psychological therapy paired with patients not referred. The interviews were analysed using a general thematic analysis.

Results

Fourteen GPs discussed 130 matched patients (65 patient pairs). Three main factors distinguished GPs' accounts of the patients they referred compared with the matched patients they did not refer. These factors were: patient initiative in requesting or showing interest in referral; estimated capacity of the patient to benefit from psychological therapy; and the GP's own capacity to help the patient in terms of skills, expertise, and time.

\section{Conclusion}

GPs gave accounts of themselves acting as rational decision makers, judging how effective they thought a referral would be based on a patient's clinical presentation and motivation, compared with the GPs' own ability to help.

\section{Keywords}

decision making; mental health; primary care; qualitative research; referrals.

\section{INTRODUCTION}

Psychological therapies are effective for common mental health problems such as anxiety disorders and depression. ${ }^{1,2}$ Patients favour psychological over drug therapies ${ }^{3}$ and have become more likely to request treatment. In addition, in the UK, the NHS patient choice agenda explicitly encourages patient influence over choice of treatment. ${ }^{4}$ However, access to psychological therapies is limited. ${ }^{5}$ GPs, in their gatekeeper role, face difficult decisions as to whom to refer for psychological therapies. Little is known about this decision-making process.

Quantitative research on referrals has identified patient, practice, and doctor characteristics associated with variation in referral rates, including referrals to mental health services. ${ }^{6-9}$ Qualitative research has explored doctors' views about, and developed models of, the factors involved in decision making regarding referrals. ${ }^{10-15}$ Patient involvement in decision making about referrals has also been studied. ${ }^{16}$ This literature may also be relevant for decision making regarding referral to psychological therapies; however, only one study ${ }^{17}$ was found that focused specifically on factors involved in GPs' referral decisions for psychological therapy, beyond patient diagnosis and problems presented..$^{18-20}$

S Stavrou, D Clin Psych, clinical psychologist, NHS Haringey; J Cape, PhD, head of psychology, Camden and Islington NHS Foundation Trust; C Barker, $\mathrm{PhD}$, reader in clinical psychology, Research Department of Clinical, Educational and Health Psychology, University College London, London.

Address for correspondence John Cape, Camden and Islington NHS Foundation Trust, St Pancras Hospital, 4 St Pancras Way, London NW1 0PE. Email: j.cape@ucl.ac.uk

Submitted: 15 February 2009; Editor's response: 2 April 2009; final acceptance: 2 July 2009.

(C) British Journal of General Practice

This is the full-length article of an abridged version published in print. Cite this article as: Br J Gen Pract 2009; DOI: 10.3399/bjgp09X454089. 
The present qualitative study used a novel procedure of asking GPs to compare decision making for matched pairs of patients: one patient who was referred and one who was not referred. This was to explore decisions about referrals for psychological therapy.

\section{METHOD}

\section{Setting}

GPs from two inner London boroughs in practices that had both practice counsellors and attached clinical psychologists were approached for inclusion. Of 30 GPs approached, 14 agreed to participate.

\section{Matched-pair interviews}

Prior to each GP interview, the attached clinical psychologists and practice counsellors identified the doctor's five most recent referrals. Criteria for the selection of patients included being between the ages of 18 and 65 years, and having been referred for psychological therapy for anxiety, depression, or both. GPs were asked to pair these cases with patients with comparable problems (in terms of type of presenting problem or diagnosis) and of the same sex and similar age. These were patients they had seen recently and had decided not to refer for psychological therapy or to mental health services.

The interview schedule consisted of asking GPs to describe briefly each patient pair and then to compare and contrast the two patients. The aim of this was to generate detailed information about what had influenced their referral decision in either case. Prompts were used for eliciting specific material identified in the literature review as being pertinent to a GP's decision making; for example, doctor-patient relationship, previous treatment, and GP's experience of similar cases. GPs had access to their electronic patient records during the interview to aid recall.

Interviews were conducted by the first author: a trainee clinical psychologist at the time of the study. The interviews lasted approximately 50 minutes and were recorded and later transcribed.

\section{Data analysis}

A general thematic analysis was used to identify dominant themes from the transcripts..$^{21,22}$ The first stage of the analysis involved a close reading of each GP's transcript to identify the main themes across the patient pairs. The second stage involved a cross-case analysis, which identified common themes across the whole sample of 65 patient pairs. The final stage involved grouping these emerging themes into higherorder categories. The process was iterative, with the themes emerging from the analysis of the first few GPs' interviews being re-examined during analysis of subsequent transcripts. The analysis was principally

\section{How this fits in}

With limited access to psychological therapies, GPs face difficult decisions as to whom to refer. Little is known about how they make these decisions. This study found that GPs described themselves as considering a patient's wishes for, and interest in, referral; the patient's capacity to benefit from psychological therapy; and their own capacity to help the patient. They balanced likely effectiveness of psychological therapy for each patient with their own ability to help.

conducted by the first author. However, all transcripts were closely read by the second author to ascertain the plausibility of the analysis. The third author commented on the overall structure of the themes.

To establish responder validity, each GP was sent a list of the main themes from the analysis of his or her interview and was asked to rate on 5-point scales how accurate, clear, and complete these themes were. GPs were also asked to identify any errors or omissions. Eight of the $14 \mathrm{GPs}$ responded; no further attempt was made to contact the six non-responders. GPs were generally in agreement with the analysis of their transcripts, all giving it ratings of 4 or 5 on the three scales. Two commented on the wording of their summarised themes (that is, they made a distinction between 'sincerity' and 'commitment' in patients' requests for referrals). These individual themes were modified slightly in accordance with GPs' comments, but this did not affect the overall structure of the aggregated themes.

\section{RESULTS \\ Participant characteristics and patient matching}

Seven male and seven female GPs participated (mean age 39 years, range 30-55 years; mean 11 years in practice, range 2-30 years). Most worked in larger group practices and had an interest in mental health problems. Five matched-patient pairs were discussed with nine of the participating GPs, with only four pairs being used with five GPs as a result of interview time constraints (the four included pairs were the four most recently referred). Thus 65 patient pairs (130 individual cases) were discussed in total. Table 1 gives the GP

Table 1. GP classification of problems of the 65 patient pairs (referred and non-referred).

\begin{tabular}{lc} 
Problem & $n$ \\
\hline Depression & 28 \\
\hline Anxiety (mixed or unspecified) & 7 \\
\hline Panic disorder & 6 \\
\hline Obsessive compulsive disorder & 2 \\
\hline Mixed depression and anxiety & 22 \\
\hline
\end{tabular}


diagnoses of the 65 patient pairs. There were 38 female and 10 male pairs, with 17 pairs being unmatched for sex. Age matching was less satisfactory, with only 18 pairs being matched within 5 years (missing data on one or both of 13 pairs).

\section{Themes that distinguished referred and non-referred patients}

Three superordinate themes distinguished patients who were referred and the matched patients who were not referred: the patient's request and interest in referral; the patient's likely benefit from psychological therapy; and the GP's perceived capacity to help (Table 2). Although these are presented as discrete themes, in reality they were all interrelated. Often a GP would be prompted to make a referral due to a combination of two or three factors, with individual GPs placing emphasis on some factors more than others.

GPs commented frequently about waiting lists when considering whether to refer a patient or not. Talking therapies were viewed as a valuable resource and GPs would prioritise patients whom they felt would use the referral well and benefit most.

This background context around their decision making permeated the interviews, both in discussing influences broadly on referral decisions and when discussing specific patient pair comparisons.

\section{Patient initiative}

Patient request for help. Many referred patients explicitly asked their GP for additional help with their problems. This request was either a direct request for referral or a request for something more to be done. At times, the patient's request for a referral was at the instigation of a third party (family member, hospital consultant, or occupational health doctor). No GP refused a patient a referral if the patient asked for it:

'I feel that the most probable reason why I referred him on was that he asked for it.' (GP 1, patient pair 5)

Requests for referral reassured GPs, in that the patient had thought about it beforehand, possibly read up on different types of psychological therapies and showed motivation:

'I talked to her about what she wanted to do and she said that she would like to see a counsellor ...

I think if someone made an appointment themselves, came in with quite a good understanding of what things might help, then I am probably more likely to discuss referring them on.' (GP 8, patient pair 38)

A patient asking for a referral also gave a focus to the consultation and as a result saved on consultation time:

'This man asked for a referral, which is a common reason why I refer someone. If they come in here and say, "I want to be referred to a counsellor", why waste time? I will explore it a little and then I will refer them.' (GP 9, patient pair 44)

Patient not interested in referral. Other patients did not wish to be referred or the GP felt that some patients would not be interested and had never discussed it with them. Some patients had not found

\section{Table 2. Themes distinguishing referred and non-referred patients.}

Theme + - $^{\mathrm{a}}$

Prevalence $^{\mathrm{b}}$

1. Patient request to be referred versus not interested in referral Requested referral/help +

2. Patient likely to benefit from psychological therapy versus unlikely to benefit

Suitable for psychological therapy (psychologically minded/insightful) +

Not psychologically minded/lack of insight -

Specific clinical problem treatable by psychological therapy +

GP felt nothing to be gained from referral -

Reliable with appointments +

Patient unreliable with appointments -

Patient unreliable with appointments -

3. GP lacks capacity to help versus has capacity to help

GP lacks expertise/time/skills +

GP able to treat patient/has skills -

Severity of problem +

Multiple minor problems -

Patient not improving +

Patient improving -

${ }^{a}+$ reason to refer, - reason not to refer. ${ }^{b}$ Prevalence of themes across GPs: typical (seven or more GPs), variable (three to six GPS), uncommon (one or two GPS). 
psychological therapy useful in the past or simply could not see how it could benefit them:

'She has a history of anxiety and depression and she has seen a counsellor in the past, which she didn't find particularly helpful.' (GP 2, patient pair 7)

'He is the sort who if you asked "What do you think about going to see a counsellor?" would say "What, do you think I am mad or something?" $\mathrm{He}$ is slightly old fashioned in that way.' (GP 7, patient pair 35)

GPs reported that some patients felt happy with just seeing a GP and were not asking for more to be done:

'She is happy to see me. I may have even asked if there was anything else I could do to help, in a sort of roundabout way, but she doesn't come up with anything like "Please refer me to the counsellor."' (GP 3, patient pair 14)

\section{Patient likely to benefit from psychological therapy}

Patient suitability. A GP's decision around whether or not to refer a patient, frequently centred on how suitable a candidate the GP felt the patient was for psychological therapies. Some patients referred by GPs were considered to be more psychologically minded and more motivated to talk about their problems, and were described by GPs as having more insight:

'He was someone who I feel that would relate well to psychology because of the way he talked about his problems ... He related them. He saw why he was having his problems.' (GP 6, patient pair 25)

'She is someone who might do well from having psychology input in that she wants it, she is motivated, she is articulate and probably could reflect on various things.' (GP 8, patient pair 37)

Other patient characteristics that some GPs considered relevant when referring a patient for psychological therapy included a certain level of intelligence, a reasonable level of education and the ability to learn new skills and appreciate goals:

'He is quite an intelligent man and I thought that he would be quite suitable because he had time to come and he had some knowledge of CBT and I felt he could be helped by it.' (GP 2, patient pair 6)
I think there is probably a type of individual who particularly fit ... who [has] a certain amount of education, say so they can appreciate goals, appreciate objectives, look at things in a structured way, doing the homework, taking all that on board.' (GP 11, patient pair 53)

Conversely, many non-referred patients were described by their GPs as 'lacking insight' or 'not very psychologically minded'. These patients would often present to the GP complaining of physical symptoms; for example, headaches, back aches, or palpitations:

'She does feel that the anxiety is a problem, but she feels that it is tension in her muscles ... physical. Because she has quite clear views about it I don't think she would benefit.' (GP 10, patient pair 46)

'She comes in saying that her life is dreadful, but she isn't depressed. She has a lot of anxiety problems, but she is not mentally ill. Somehow I just don't feel that she would benefit ... she wouldn't get that much out of it. She has had multiple physical investigations, but her symptoms are the same. I do try to think psychologically with these patients and try to reflect back ... "Do you think depression, anxiety or pressure in looking after your family are part of it?", and she says no each time.' (GP 3, patient pair 12)

Specific clinical presentation. Certain patient presentations directly influenced a GP's decision to refer. For example, panic disorder and obsessive compulsive disorder (OCD) were described as especially suitable for treatment with cognitive behavioural therapy (CBT):

I do feel that it [panic] is one of those things that is incredibly treatable not using medication. It is much more clear cut, for example, than when I see a depressed patient. I have a lower threshold for panic attacks ... I have a slight reflex action in terms of thinking "Oh, panic attacks. I know someone who can sort this out."' (GP 10, patient pair 47)

'She described how she was having problems washing hands, compulsive behaviour that had been going on for a long time ... and so on the background of it being a specific problem, like OCD, I thought well this might be someone that might benefit a lot from seeing a psychologist than seeing a GP.' (GP 8, patient pair 40) 
Patient reliability. Most GPs viewed counselling and psychology as a valuable resource, and would judge a patient's motivation when considering a referral. Patients who had a history of not attending, either with their own GP or other specialists to whom they had been referred, were considered unreliable and commonly cited as a reason against referral:

'She is someone who is notorious about 'DNAing' and is quite unreliable ... she is also the kind of person who if she received a letter saying that she would have to wait another 6 to 8 weeks, she probably wouldn't be interested ... you only really want to refer someone who you think will benefit and turn up.' (GP 2, patient pair 8)

'She doesn't come very often, not very committed I think, a few DNAs. I think if you're not going to show commitment to see your GP regularly you certainly aren't going to go see a counsellor regularly.' (GP 5, patient pair 25)

Patient would not benefit. Some GPs felt that it was 'a GP's lot' to see patients with intractable and chronic problems, who in their eyes would not benefit from a referral. These patients on the whole had multiple, minor physical health complaints and were not necessarily improving with the GP's treatment. So, whereas chronicity and lack of improvement were in many cases seen as factors promoting referral with these patients (see following section on GPs' capacity to help), GPs counted them as factors against referring due to the perceived lack of benefit or clear outcome from the referral:

'This lady I have been seeing for years. A lot of physical illness, asthma, blood pressure and I think as the years go by, mildly clinically depressed. I have her on 'ADs', but I am not sure whether they have made much of a difference ... whenever I see her and ask how she is, she always tells me how terrible things are and how depressed she is ... I don't think I have ever suggested counselling or anything for her ... it was a very slow history, no specific anxiety or acute symptoms ... if I don't feel that there is going to be any relatively quick outcome then that does sort of stop me thinking about referring.' (GP 3, patient pair 15)

'I suppose the patient I didn't refer you could describe as a bit of a heartsink patient who I have tried various strategies with over the years and none of them really work, and I think I have slightly accepted ... OK ... I'll offer this supporting treatment.' (GP 10, patient pair 46)

\section{GPs' capacity to help}

GP expertise/time/skills. GPs stated that they did not feel they had the skills to help certain patients with their problems, as in cases of childhood sexual abuse, OCD, or post-traumatic stress disorder:

'I didn't know what to do with somebody who has been abused. I could just listen, but that is a bit like a GP trying to treat a heart attack. It's best with the specialists.' (GP 4, patient pair 18)

For some referred patients, GPs would also comment on just 'feeling that the patient needed more' than they felt they could offer him or her, without specifying exactly what they felt they could not provide:

'I have to say with her it felt like there were an awful lot of underlying things. I think I felt she needed more than I could give.' (GP 10, patient pair 46)

Another factor was not having enough time and knowing that a counsellor or psychologist would be able to offer much longer appointments:

'I don't quite know what makes her tick ... she seems OK when I see her and we talk about practical issues like relationships, but I get a different story from her mother. So, I feel that I don't really know what is going on ... and maybe the psychologist could spend 50 minutes whereas I can spend just 10 minutes.' (GP 3, patient pair 12)

I wanted him to talk things through in a much more detailed way than I was able to provide. The counsellors have much longer than my consultations. I very rarely take double consultations unless it's a measure of last resort.' (GP 13, patient pair 54)

With other patients, many GPs felt they did not need to refer as they were able to offer their patients something themselves. Often this was due to the patient responding well to the medication that the GP had prescribed, and seemed to be doing 'OK' or improving with the GP's treatment:

'I felt I was offering something. She responded well to medication and she is also easier to talk to. The time we spent together we covered a lot of things and she accepted the mechanisms behind her depression ... how the treatment was going to work.' (GP 1, patient pair 3) 
'I think the main reason why I didn't refer him on was because he seemed to respond well, usually, to the amount of support I was able to give ... I felt that I was able to manage that situation pretty well myself.' (GP 6, patient pair 28)

GPs also cited feeling able to manage a patient with the skills they had as a reason not to refer on. GPs' confidence that they were able to provide appropriate care also played a role:

'She is within my gamut. I can sort of keep her moderately ticking over. She isn't acting out or worrying me in any way. She is not spilling it over onto anybody else, she is a contained depressive and I suppose I feel I have enough skills up my sleeve.' (GP 7, patient pair 32)

'The way we had been managing it was a combination of her taking time off work and actually just seeing her or speaking to her on the phone to see how things were going. I didn't refer because it felt like something that was solvable.' (GP 10, patient pair 47)

Severity of presentation. The more severe the presentation, the more likely a GP would consider referral. Severity appeared to be judged on various levels. GPs mentioned the extent to which the patients' problems were interfering with their everyday lives, especially work. The frequency with which they presented to the GP within a short time period was another indicator of severity, and also how distressed they were in the consultation with the GP:

'She was coming to me several times a week and was very distraught. I think the severity ... also she normally works and was OK there ... everything seemed to be going to pieces and made me feel like I should refer.' (GP 10, patient pair 47)

Conversely, GPs were less likely to refer patients whose problems were less severe, clearly linked to a stressful life event, or which appeared to be transient. These problems made much more sense to GPs and possibly improved the doctor-patient relationship, aiding the patient's recovery:

'This lady presented with some major life events: a death, problems in her marriage, problems at home ... with her I felt that it was something that I could deal with. You know, I could most probably relate to the things that had happened to her in life ... I felt it was easier to cope with, to deal with a patient like that.' (GP 1, patient pair 3)
Lack of improvement/improving. When patients were not improving with the GP's treatment, they would more likely be referred:

'She had quite an acute depressive episode where she felt she just couldn't cope. High workload, couldn't cope, went off sick, came to see me and it's really gone on from there. She, if anything, has gone downhill I would say. She hasn't improved on medication.' (GP 9, patient pair 42)

'This lady had a history of anxiety and depression and she has been on antidepressants for a long time and she felt that they weren't working.' (GP 13, patient pair 61)

On the other hand, a common reason stated by GPs for not referring on was that the patient was doing OK or showing signs of improvement with the GP's treatment. This was linked with the patient seeming happy with what the GP was offering him or her, and not pushing for a referral:

'I didn't refer this patient as I felt that she did well ... she responded well to the antidepressants and I think that it is actually quite a positive thing when somebody comes back and says they are feeling a bit better. It made me feel better about carrying on seeing her.' (GP 1, patient pair 1)

'He was seen a number of times here and carried on coming but seemed to do quite well with the medicines. I suppose that might be why I didn't feel the need to use anyone else because he seemed to have some improvement.' (GP 6, patient pair 26)

\section{Interaction between themes in decision making}

The three major themes interacted in that GPs' accounts of their decision making generally included more than one theme and more than one factor within a theme. GPs would make a referral based on a combination of a few factors. For example, in the following extract, the referral was based primarily on the patient not improving, the GP lacking time, and the problem (somatisation) being treatable by psychological therapy:

'I felt that the somatic symptoms he had and the somatisation he was portraying was just quite severe. I didn't want him to keep coming back to me every time he had any little symptom about that because in our 10-minute appointments it isn't really an effective way of dealing with that 
and going through the whole "Well, do you think it's because you are stressed at the minute?", or whatever. And so I referred him for help in dealing with that specific problem, which I thought would probably, I hope, do well with some psychology.' (GP 6, patient pair 27)

Individual GPs placed emphasis on some factors more than others. For example, one GP regularly described basing referral decisions on a patient's personal characteristics (level of intelligence and reliability) and on whether as a GP he felt able to help:

'He is quite an intelligent man and I thought that he would be quite suitable, because he had the time to come (which is obviously important as appointments are during the day) ... I will pair him with a gentleman, who I don't think he is particularly compliant with his appointments ... also he was someone with whom I had quite a good relationship with and I felt that we could chat about things. He is also on medication and seems to be doing reasonably well, so I didn't think at this stage that he was someone who I necessarily needed to refer.' (GP 2, patient pair 6)

\section{DISCUSSION}

\section{Summary of main findings}

Three main factors distinguished GPs' accounts of the patients they referred compared with the matched patients they did not refer. These factors were: patient initiative in requesting or showing interest in referral; estimated capacity of the patient to benefit from psychological therapy; and the GP's own capacity to help the patient in terms of skills, expertise and time. These three factors interacted, so GPs would generally report deciding to make a referral or not based on a combination of two or three factors. Individual GPs placed emphasis on some factors more than others. However, overall, the accounts they gave were of acting as rational decision makers, balancing patient choice and likely effectiveness of psychological therapy with their own ability to help. Finally, waiting lists were constantly at the back of many GPs' minds when thinking about referrals.

\section{Strengths and limitations of the study}

The study design which asked about recent referral decisions, allowed GP decisions to be examined in greater detail than simply interviewing doctors about their decision making in general. The novel matchedpatient design increased salience of key factors involved in decisions by constant comparison of referred and non-referred patients. The study also increased responder validity by feeding back a summary of factors for and against referring patients to each GP following the analysis of his or her interview. These methods all followed good-practice guidelines for qualitative research. ${ }^{23}$

Limitations include that the GP participants were likely to be more interested in mental health and hence the findings may not be generalisable to all GPs. The retrospective recall element may have introduced memory biases, although the GPs had their electronic patient notes available during the interviews to aid recall. The GPs were asked to identify the non-referred sample of patients themselves and it is likely that they chose patients they remembered well, or those where they felt they had made a good decision not to refer on. This may have left more ambiguous or problematic complex cases unexamined. In their accounts, GPs may have chosen reasons for referring and not referring to present themselves in a good light to the interviewer - as rational, 'good' decision makers - rather than identifying more emotional, less rational factors. The method of asking GPs to contrast their referral choices is likely to prompt more rational, conscious reasons. The method is less suited to eliciting emotional factors that may also have motivated GPs. The matched-patient design by its nature limits the extent to which the factors used to match patients (diagnosis, age, sex) would appear in GP accounts as reasons for or against referral. Additionally, while matching of referred and non-referred patients on type of problem and sex was good, matching for age was less satisfactory

\section{Comparison with existing literature}

GPs' decision making about referrals for psychological therapies in the present study was influenced by a broad range of factors: patient, problem, doctor, and contextual. This reflects the general literature on medical decision making, including studies of referrals, that a range of type of factors influence decisions..$^{10,14,24}$

Patient request for referral has been identified as a key factor in prior studies of GP referrals to mental health and other specialists. ${ }^{11,13,17,25-27}$ The impact of patient requests and interest has also been found important in decisions as to whether to prescribe antidepressant medication. ${ }^{28-30}$ Beyond mental health, the literature on shared decision making across medicine highlights the increasing role of patient initiative and involvement in medical decision making. ${ }^{31,32}$

Previously, a specific type of patient's presenting problem or diagnosis was found to be important in GP referral decisions between types of psychological therapy and mental health service. ${ }^{18-20}$ Severity of depression was found to predict referral in a recent study investigating treatment decisions in relation to 
scores on depression severity questionnaires. (The present study was carried out prior to the widespread introduction of depression questionnaires in general practice. $)^{33}$ Patient insight and ability to articulate problems and GPs' feeling that they have nothing more to offer a patient - both identified by GPs in the present study as reasons for referring patients for psychological therapy - were also found important in the one previous study of referral decisions for psychological therapy. ${ }^{17}$

Patient intelligence, ability to articulate problems, and insight relate to psychological mindedness, which predicts benefit from psychological therapy. ${ }^{34}$ However, selection for psychological therapy on the basis of these characteristics has also been criticised on equity grounds, as likely to impede access to social groups who referrers and assessing psychological therapists deem to lack these characteristics. ${ }^{35}$ Given the sensitivity of this area, how GPs assess intelligence and insight in the context of social and cultural differences is an important area of further study.

Rational reasons for referral to mental health services with a specific reason in mind have been distinguished from reactive emotional reasons in response to the GP feeling unable to manage the patient. ${ }^{15}$ In the present study, even where GPs gave reasons of lacking capacity to help the patient, this only led to referral if they also thought the patient would benefit from psychological therapy. Patients whom the GPs considered could not be helped by anyone were found entirely among those not referred.

In the general decision-making literature, optimising procedures, where all factors are considered to locate the best solution, have been contrasted with 'satisficing' procedures, where the decision maker stops searching for more information once he or she has a 'good enough' solution. ${ }^{36}$ Satisficing is a reasonable strategy where there are time constraints, as in general practice. The limited information from the present study suggests that GPs were using satisficing strategies to make their referral decisions.

\section{Implications for future research and clinical practice}

While the matched-patient design of this study has advantages over previous designs in identifying factors related to actual GP referral decisions, the method remains subject to post hoc justification of decisions and, as with much GP research, ${ }^{37}$ is crosssectional rather than longitudinal. Decisions about whether to refer or not are often made over several consultations. Methods of tracking GPs' thinking about management longitudinally over successive consultations, including whether to refer, are needed.
Clinical decision making in the context of limited access to beneficial treatments has been described as an issue of the heart as well as the mind ${ }^{38}$ In their decision making about referrals for psychological therapy, the GPs in this study described themselves as struggling to make decisions for the benefit of their patients with awareness of limited access and long waiting lists for psychological therapies. Their weighting of different criteria varied, as did their views of their own skills in being able to help patients compared with a psychological therapy referral. While variation will be inevitable, GPs can be assisted in their decision making by: good information about relative effectiveness of psychological therapies compared with GP treatment as usual; ${ }^{1}$ confidence in their own capacity to help many patients; ${ }^{39}$ and approaches, within the limits of general practice, to support the patients for whom effective treatments are not available. ${ }^{40}$

Referral decision making is always within a local context of intervention and service referral options and constraints. The development of new types of psychological intervention, such as guided self-help ${ }^{41}$ and computerised $\mathrm{CBT}^{42}$ and new systems, such as the UK Improving Access to Psychological Therapies services, ${ }^{43}$ bring new contexts and decision-making requirements. Stepped-care models ${ }^{44}$ seek to bring coherence and a logical framework to design of care pathways and decision-making steps. GP decision making within stepped-care systems for common mental health problems will be an important future direction of research.

\section{Ethical approval}

Ethical approval was obtained from Camden and Islington Community Local Research Ethics Committee

\section{Competing interests}

The authors have stated that there are none

\section{Acknowledgements}

We would like to thank the GPs who participated.

\section{Discuss this article}

Contribute and read comments about this article on the Discussion Forum: http://www.rcgp.org.uk/bjgp-discuss

\section{REFERENCES}

1. National Institute for Clinical Excellence. Depression: management of depression in primary and secondary care. London: NICE, 2004.

2. Roth A, Fonagy P. What works for whom? A critical review of psychotherapy research. 2nd edn. New York: Guilford, 2005.

3. Priest RG, Vize C, Roberts A, et al. Lay people's attitudes to treatment of depression: results of opinion poll for Defeat Depression Campaign just before its launch. BMJ 1996; 313(7061): 858-859.

4. Department of Health. The NHS plan: a plan for investment, a plan for reform. London: HMSO, 2000.

5. Layard R. The case for psychological treatment centres. BMJ 2006; 332(7548): 1030-1032.

6. Creed F, Gowrisunkur J, Russell E, et al. General practitioner referral rates to district psychiatry and psychology services. Br J Gen Pract 1990; 40(340): 450-454.

7. O'Donnell CA. Variation in GP referral rates: what can we learn from the literature? Fam Pract 2000; 17(6): 462-471.

8. Verhaak PFM. Analysis of referrals of mental health problems by 


\section{COMMENTARY}

\section{GPs' views on their handling of depression}

The irresistible rise of the selective serotonin reuptake inhibitor is a fact of contemporary life; antidepressant prescription volumes and costs have more than trebled since their introduction in the early 1990s. ${ }^{1}$ There have been other changes too: mental illness can be stigmatising, but with around 30 million prescriptions for antidepressants per year in England and Wales, it could be argued that the medical treatment of depression has been normalised. This phenomenon is not unique to the UK. The view from over here is that the US is the home of widely available and interminable psychotherapy; Americans have seen a similar rise in prescriptions for antidepressants while rates of psychotherapy have remained flat or even declined over the last 20 years. ${ }^{2}$

Two papers in this issue of the BJGP explore GPs' views on two important aspects of this change in prescribing; Macdonald et al ask GPs in Scotland why they think it has happened; ${ }^{3}$ Stavrou et al ask GPs in London who they refer for psychological therapy and why. ${ }^{4}$ GPs' views are important because we do most of the prescribing of antidepressants. We also have some influence on whom is referred for psychotherapy; the government has made a commitment to increasing availability of psychological interventions in primary care, but psychotherapy is still a carefully rationed resource.

There is something slightly disconcerting about reading Macdonald et al's summary of their interviews with 63 GPs. Many of the explanations offered by GPs for the increase in prescribing are thoughtful and insightful. The usual suspects are lined up: The Defeat Depression Campaign, the pharmaceutical industry, the consumerist attitude of the patient with high expectations of happiness, and a pill for social ills. The causes of the increase are largely seen to be external; as the authors comment 'GPs did not see themselves as drivers of change'. There is some soul searching on the medicalisation of misery, but the prevailing view seems to be that this is an almost inevitable consequence of our limited resources and the pressures we face to solve social problems.

Stavrou et al found their inner London GPs to be altogether more confident in deciding whom to refer and not to refer for psychotherapy. Fourteen GPs discussed pairs of patients: one referred and one not referred. The plausible picture that emerges is largely one of rational decision makers, acting in concert with their patients' wishes. As the authors point out, this may reflect a bias, in that the GPs selected the patients they wished to discuss. Areas of potential uncertainty or difficulty do not appear to have been explored by the participants. For example, 'non-psychologically minded' patients with 'multiple minor physical health complaints' who were 'not necessarily improving with the GP's treatment' were still considered unsuitable for psychological therapy; whatever happened to the reattribution of minor somatic symptoms?

These papers address two important and not unrelated questions: how did we come to be prescribing so many antidepressants; and how do we decide who will benefit from referral to psychotherapy? If we want to answer these questions we need to use other methodologies to look at patient outcomes. The missing piece of the puzzle in any discussion of GPs' selection of patients for referral to psychotherapy is evidence for predictors of response to treatment. This is currently lacking. And even more surprising is the fact that we bewail the increase in antidepressant prescribing without knowing very much about its impact.

\section{David Kessler,}

Primary Health Care, University of Bristol, 25 Belgrave Road, Bristol BS8 2AA. E-mail: david.kessler@bristol.ac.uk

\section{Provenance}

Commissioned; not peer reviewed.

\section{REFERENCES}

1. Hollinghurst S, Kessler D, Peters TJ, Gunnell D. Opportunity cost of antidepressant prescribing in England: analysis of routine data. BMJ 2005; 303: 999-1000.

2. Olfson M, Marcuss S, Druss B, et al. National trends in the outpatient treatment of depression. JAMA 2002; 287: 203-209.

3. Macdonald S, Morrison J, Maxwell M, et al. 'A coal face option': GPs' perspectives on the rise in antidepressant prescribing. Br J Gen Pract 2009; DOI: 10.3399/bjgp09X454106

4. Stavrou S, Cape J, Barker C. Decisions about referrals for psychological therapies: a matched-patient qualitative study. Br J Gen Pract 2009; DOI: 10.3399/bjgp09X454089

\section{DOI: 10.3399/bjgp09X454098}

general practitioners. Br J Gen Pract 1993; 43(370): 203-208

9. O'Sullivan C, Omar RZ, Ambler G, et al. Case-mix and variation in specialist referrals in general practice. Br J Gen Pract 2005; 55(519): 529-533.

10. Bailey J, King N, Newton P. Analysing general practitioners' referral decisions. II. Applying the analytical framework: do high and low referrers differ in factors influencing their referral decisions? Fam Pract 1994; 11(1): 9-14.

11. Morgan D. Psychiatric cases: an ethnography of the referral process. Psychol Med 1989; 19(3): 743-753.

12. Dowie R. General practitioners and consultants: a study of outpatient referrals. London: King's Fund, 1983.

13. Evans A. A study of the referral decision in general practice. Fam Pract
1993; 10(2): 104-110.

14. Newton J, Hayes V, Hutchinson A. Factors influencing general practitioners' referral decisions. Fam Pract 1991; 8(4): 308-313.

15. Nandy S, Chalmers-Watson C, Gantley M, et al. Referral for minor mental illness: a qualitative study. Br J Gen Pract 2001; 51(467): 461-465.

16. Jones IR, Berney L, Kelly M, et al. Is patient involvement possible when decisions involve scarce resources? A qualitative study of decision-making in primary care. Soc Sci Med 2004; 59(1): 93-102.

17. Sigel P, Leiper R. GP views of their management and referral of psychological problems: a qualitative study. Psychol Psychother 2004; 77(Pt 3): 279-295.

18. Corney RH. Mental health services in primary care: the overlap in 
professional roles. J Ment Health 1999; 8(2): 187-194.

19. Cape J, Parham A. Rated casemix of general practitioner referrals to practice counsellors and clinical psychologists: a retrospective survey of a year's caseload. Br J Med Psychol 2001; 74(Pt 2): 237-246.

20. Pilgrim D, Rogers A, Clarke S, et al. Entering psychological treatment: decision-making factors for GPs and service users. J Interprof Care 1997; 11: 313-23.

21. Barker C, Pistrang N, Elliot R. Research methods in clinical psychology: an introduction for students and practitioners. 2 nd edn. Chichester: Wiley, 2002.

22. Boyatzis RE. Transforming qualitative information: thematic analysis and code development. London: Sage, 1998.

23. Mays N, Pope C. Qualitative research in health care: assessing quality in qualitative research. BMJ 2000; 320(7226): 50-52.

24. Chapman G, Sonnenberg F (eds). Decision making in health care theory, psychology, and applications. Cambridge: Cambridge University Press, 2000.

25. Kravitz RL, Bell RA, Azari R, et al. Direct observation of requests for clinical services in office practice: what do patients want and do they get it? Arch Intern Med 2003; 163(14): 1673-1681.

26. Kravitz RL, Franks P, Feldman M, et al. What drives referral from primary care physicians to mental health specialists? A randomized trial using actors portraying depressive symptoms. J Gen Intern Med 2006; 21(6): 584-589.

27. Robertson NC. Variations in referral pattern to the psychiatric services by general practitioners. Psychol Med 1979; 9(2): 355-364.

28. Kravitz RL, Epstein RM, Feldman MD, et al. Influence of patients' requests for direct-to-consumer advertised antidepressants: a randomized controlled trial. JAMA 2005; 293(16): 1995-2002.

29. Hyde J, Calnan M, Prior L, et al. A qualitative study exploring how GPs decide to prescribe antidepressants. Br J Gen Pract 2005; 55(519): 755-762.

30. Kendrick T, King F, Albertella L, et al. GP treatment decisions for patients with depression. Br J Gen Pract 2005; 55(513): 280-286.

31. Edwards M, Davies M, Edwards A. What are the external influences on information exchange and shared decision-making in healthcare consultations: a meta-synthesis of the literature. Patient Educ Couns 2009; 75(1): 37-52.

32. Edwards A, Elwyn G. Shared decision making in health care: achieving evidence-based patient choice. 2nd edn. Oxford: Oxford University
Press, 2009.

33. Kendrick T, Dowrick C, McBride A, et al. Management of depression in UK general practice in relation to scores on depression severity questionnaires: analysis of medical record data. BMJ 2009; 338: b750. doi: $10.1136 /$ bmj.b750.

34. McCallum M, Piper W. Psychological mindedness. Psychiatry 1996; 59(1): 48-64.

35. Lawson R, Guite H. Psychological therapies for common mental illness: how effective and equitable is provision? Prim Care Ment Health 2005; 3(1): 5-12.

36. Newell BR, Lagnado DA, Shanks DR. Straight choices: the psychology of decision making. Hove, UK: Psychology Press, 2007.

37. Kessler D, Bennewith O, Lewis G, et al. Detection of depression and anxiety in primary care: follow up study. $B M J 2002 ; 325(7371)$ : 1016-1017.

38. Sabin JE. The second phase of priority setting: fairness as a problem of love and the heart: a clinician's perspective on priority setting. BMJ 1998; 317(7164): 1002-1004.

39. Cape J, Barker C, Buszewicz M, et al. General practitioner psychological management of common emotional problems (I): definitions and literature review. Br J Gen Pract 2000; 50(453): 313-318.

40. Mathers NJ, Gask L. Surviving the 'heartsink' experience. Fam Pract 1995; 12(2): 176-183.

41. Gellatly J, Bower P, Hennessy S, et al. What makes self-help interventions effective in the management of depressive symptoms? Meta-analysis and meta-regression. Psychol Med 2007; 37(9): 1217-1228.

42. Kaltenthaler E, Brazier J, De Nigris E, et al. Computerised cognitive behaviour therapy for depression and anxiety update: a systematic review and economic evaluation. Health Technol Assess 2006; 10(33): $1-186$

43. Department of Health, Care Services Improvement Partnership, National Institute for Mental Health in England. Improving access to psychological therapies: implementation plan: national guidelines for regional delivery. London: Central Office of Information, 2008, http://www.iapt.nhs.uk/wp-content/uploads/2009/04/nat-guidelinesregional-delivery.pdf (accessed 31 Jul 2009).

44. Bower P, Gilbody S. Stepped care in psychological therapies: access, effectiveness and efficiency: narrative literature review. Br J Psychiatry 2005; 186: 11-17. 